\title{
gु \\ Mobilities and scattering times in decoupled graphene monolayers
}

\author{
H. Schmidt, T. Lüdtke, P. Barthold, and R. J. Haug \\ Institut für Festkörperphysik, Leibniz Universität Hannover, Appelstr. 2, 30167 Hannover, Germany
}

(Received 9 February 2010; published 2 March 2010)

\begin{abstract}
Folded single-layer graphene forms a system of two decoupled monolayers being only a few angstroms apart. Using magnetotransport measurements we investigate the electronic properties of the two layers conducting in parallel. We show a method to obtain the mobilities for the individual layers despite them being jointly contacted. The mobilities in the upper layer are significantly larger than in the bottom one indicating weaker substrate influence. This is confirmed by larger transport and quantum scattering times in the top layer. Analyzing the temperature dependence of the Shubnikov-de Haas oscillations, effective masses and corresponding Fermi velocities are obtained yielding reduced values down to $66 \%$ in comparison to monolayers.
\end{abstract}

DOI: $10.1103 /$ PhysRevB.81.121403

PACS number(s): 73.22.-f, 72.80.-r

Since the discovery of stable two-dimensional crystals of carbon, ${ }^{1}$ single-layer, bilayer, and multilayer graphene systems have been studied intensely. 2,3 Monolayer graphene exhibits outstanding electronic properties including a linear dispersion relation, zero gap, a half-integer quantum Hall effect (QHE), and a Berry's phase of $\pi$. Single-crystal (SC) bilayer consisting of two Bernal stacked planes also shows an unconventional QHE, yielding plateaus at integer values, but with a double step at zero filling factor and a Berry's phase of $2 \pi r^{4,5}$ In addition to these systems samples with two twisted monolayers have been investigated using magnetotransport measurements, ${ }^{6}$ Raman spectroscopy, ${ }^{7}$ and scanning photocurrent microscopy. ${ }^{8}$ The rotational stacking fault of the two layers with respect to Bernal stacking decouples them and the upper one is screened from an applied backgate voltage. Especially when combined with a second gate on top, such a system could be a candidate to create two very closely lying electron and hole systems and could give rise to experiments recently discussed in theory. ${ }^{9-12}$ Twisted samples can be fabricated from monolayer flakes folded during the preparation process using micromechanical exfoliation. Besides this method epitaxial growth can be used to prepare graphene samples, also sometimes containing layers rotated with respect to Bernal stacking. ${ }^{13}$

Here, we report on transport measurements on twisted monolayers, produced by micromechanical exfoliation. Analyzing the cyclotron masses, mobilities, and scattering times deduced from the measurements, we find properties quite different from conventional monolayers and SC bilayer graphene.

Figure 1 shows a sketch of a two-layer sample. After peeling of pieces from natural graphite ${ }^{14}$ graphene is deposited on a silicon wafer covered with $330 \mathrm{~nm}$ of silicon dioxide. A suitable folded flake is located with the help of an optical microscope and $e$-beam lithography is used to structure the flake. A Hall-bar device is formed out of the graphene layers using plasma etching. After this, common contacts to both layers are formed by evaporating chromium and gold. The silicon back gate underneath the isolating silicon dioxide couples capacitively to the graphene. Applying back-gate voltages from -70 to $+70 \mathrm{~V}$, the density and the type of majority charge carriers in the two layers can be continuously tuned from holes to electrons.
To distinguish this system of two decoupled monolayers from a SC bilayer system, magnetotransport measurements at temperatures down to $1.5 \mathrm{~K}$ are performed. Applying perpendicular magnetic fields up to $13 \mathrm{~T}$, two sets of superimposed Shubnikov-de Haas $(\mathrm{SdH})$ oscillations are observed in the longitudinal resistance as shown in Fig. 2. Plotted over $1 / B$ two sets of equidistant minima can be separated, both exhibiting Berry's phases of $\pi$, indicating graphene monolayers. Sweeping the back-gate voltage at zero magnetic field the typical peak in the resistivity is observed indicating the charge-neutrality point. Hole doping is apparent in a shift of this peak to positive gate voltages. This could be attributed to a shift of the Fermi level due to the rotation of the layers as predicted by theory. ${ }^{15}$ In addition the effect of impurities left over from the preparation process cannot be ruled out. Figure 3(a) shows this field effect at different temperatures from 1.5 to $50 \mathrm{~K}$. Two regimes can be separated: for high voltages and thus high carrier concentrations the resistivity and conductivity, respectively, do not show any temperature dependence. In contrast to this, a decrease in the resistivity is observed for increasing temperatures close to the peak.

As shown by Morozov et al. ${ }^{16}$ for single-layer graphene, the resistivity can be separated into two parts. The first one, $\rho_{L}=1 / n e \mu^{*}$, is due to long-range scatterers and contains the carrier density $n$ and a mobility $\mu^{*}$ affected by long-range disorder. The second contribution due to short-range scatterers is $n$ independent and can be subtracted to remove nonlinear contributions to the overall conductivity at high backgate voltages. Applying this model to the here discussed twolayer system, the linearization can also be used, giving a total linearized conductivity $\sigma^{*}$ as plotted for $10 \mathrm{~K}$ in Fig. 3(a). It

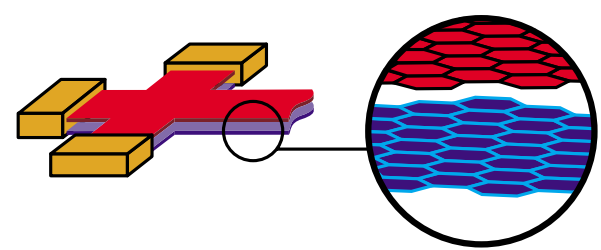

FIG. 1. (Color online) Sketch of a sample containing two graphene layers, stacked and rotated with respect to Bernal stacking. A Hall bar is etched and both layers are contacted simultaneously with chromium gold leads. 


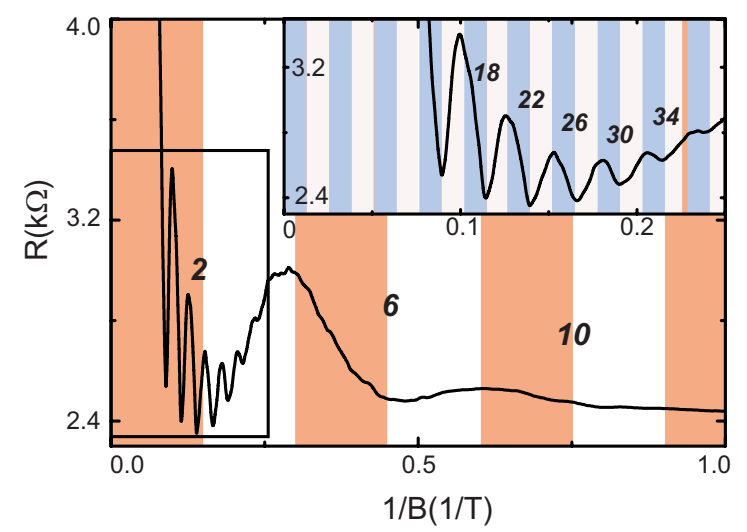

FIG. 2. (Color online) Longitudinal resistance versus $1 / B$ at a back-gate voltage of $V=70 \mathrm{~V}$ showing two superimposed Shubnikov-de Haas oscillations. The inset shows the oscillation with the higher carrier concentration and therefore belonging to the bottom layer. For both oscillations' minima, filling factors are marked and colored areas indicate the parts with negative slope of the corresponding oscillation.

is proportional to the back-gate voltage at high voltages and is the sum of the linearized conductivities of the two layers. At several fixed gate voltages $\mathrm{SdH}$ measurements were performed and the carrier concentrations $n_{1}$ and $n_{2}$ for the bottom and top layers were deduced. The results are shown in Fig. 3(b). The carrier concentration $n_{2}$ is significantly lower than $n_{1}$ due to the screening of the electric field by the bottom layer. ${ }^{6}$

To obtain the mobilities $\mu_{1}^{*}$ and $\mu_{2}^{*}$ corresponding to the part of the resistivity, which is due to long-range disorder, a model of two parallel conductors is used with the total conductivity being given by

$$
\sigma(V)^{*}=\sigma_{1}(V)^{*}+\sigma_{2}(V)^{*}=e\left[\mu_{1}^{*} n_{1}(V)+\mu_{2}^{*} n_{2}(V)\right] .
$$

Introducing $\alpha(V)$ and $C(V)$,

$$
\begin{gathered}
C(V)=\sigma(V)^{*} /\left[\operatorname{en}_{2}(V)\right], \\
\alpha(V)=n_{1}(V) / n_{2}(V),
\end{gathered}
$$

and substituting in Eq. (1) one gets a linear equation,

$$
C(V)=\mu_{1}^{*} \alpha(V)+\mu_{2}^{*}
$$

Since $\sigma(V, B=0 \mathrm{~T})^{*}, n_{1}(V)$ and $n_{2}(V)$ are deduced from the measurements, $C(V)$ and $\alpha(V)$ can be calculated. The results are shown in Figs. 3(c) and 3(d) for the hole ( $V=$ -60 to $-17 \mathrm{~V})$ and electron $(V=39-60 \mathrm{~V})$ regions, respectively. These data are in very good accordance with the expected linear behavior (2) assuming constant mobilities. The two mobilities can be directly deduced from the slope and the offset of the corresponding fit. The mobility in the bottom layer yields a value of $\mu_{1}^{*}=2600 \mathrm{~cm}^{2} / \mathrm{V} \mathrm{s}$ for holes and $3800 \mathrm{~cm}^{2} / \mathrm{V}$ s for electrons. Mobilities in the top layer exhibit larger values, being $\mu_{2}^{*}=9500 \mathrm{~cm}^{2} / \mathrm{V} \mathrm{s}$ for holes and $12300 \mathrm{~cm}^{2} / \mathrm{V}$ s for electrons. The difference between electrons and holes in both layers is attributed to the assumed doping effects. For both types of majority charge carriers, the mobility in the upper layer is significantly larger. This has to
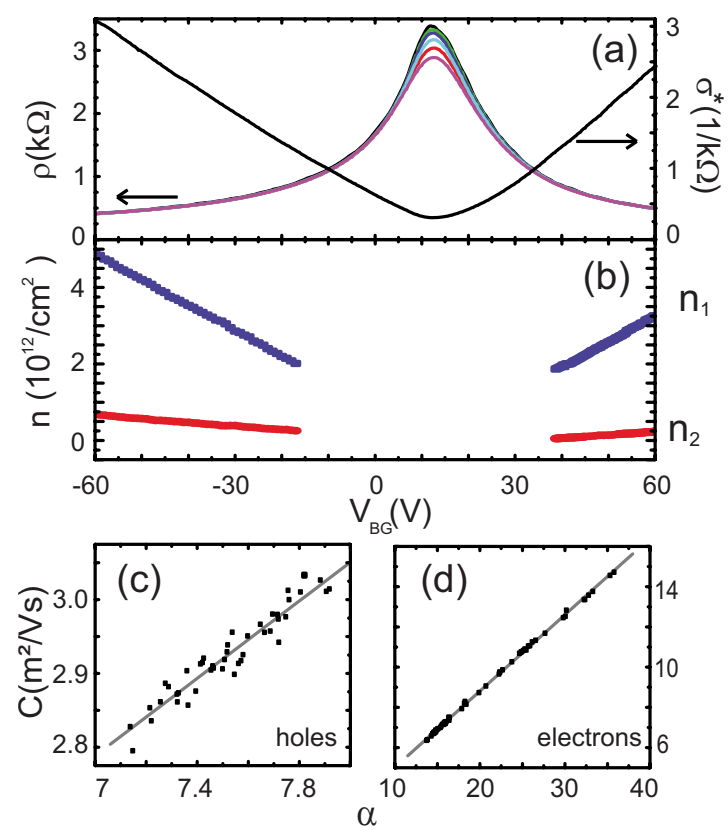

FIG. 3. (Color online) (a) Resistivity at zero magnetic field and different temperatures (from top to bottom: 1.5, 10, 15, 25, 35, and $50 \mathrm{~K})$. In addition, the linearized conductivity at $10 \mathrm{~K}$ is plotted. (b) Charge-carrier densities for $V<0$ (holes) and $V>0$ (electrons) and for both layers. From these, $\alpha$ and $C$ (see text) are calculated and plotted in (c) and (d) with corresponding linear fits (solid lines).

be explained by the fact that the second layer does not lie directly on the substrate which normally decreases the mobility of graphene due to surface charge traps, interfacial phonons, and substrate stabilized ripples. Whereas at $B$ $=0 \mathrm{~T}$ for high carrier concentrations no temperature dependence of the resistivity is observed, with applied magnetic field the temperature influences the resistivity by damping of the amplitudes in the $\mathrm{SdH}$ oscillations. Increasing the temperature $T$ the amplitudes of the oscillations decrease as shown in Fig. 4. This change in amplitude $\Delta R$ at a fixed magnetic field $B$ follows the relation $\Delta R$

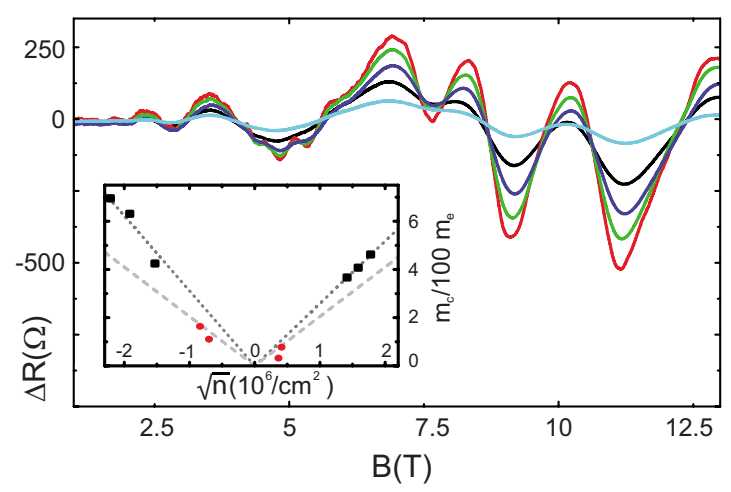

FIG. 4. (Color online) Shubnikov-de Haas oscillations at $-60 \mathrm{~V}$ back-gate voltage and different temperatures $(1.5,10,15,25$, and $35 \mathrm{~K})$. A background at $50 \mathrm{~K}$ is subtracted for better visibility. The inset shows cyclotron masses for both layers (layer 1, squares; layer 2, dots) and the values expected for a Fermi velocity of a monolayer (gray dashed lines) and the velocities fitted to our bottom layer data (black dotted line). 
$\propto(T / B) / \sinh (P T / B)$ with the parameter $P=2 \pi^{2} k_{B} m_{c} / \hbar e$ containing the cyclotron mass $m_{c}=\sqrt{h^{2} n / 4 \pi v_{F}^{2}}$ with the Fermi velocity $v_{F}$.

Fitting the equation for the amplitude to our data the effective masses at different back-gate voltages are obtained and shown in the inset of Fig. 4. Squares indicate bottom layer values and circles indicate top layer ones. These values are compared to the expected masses for a monolayer with $v_{F}=1 \times 10^{6} \mathrm{~m} / \mathrm{s}$, shown as gray dashed lines. The cyclotron masses in the upper layer seem to be in good accordance with this expectation although the superposition with the other oscillation and the low carrier concentration in this layer make it difficult to obtain the exact amplitude. The values for the bottom layer show the expected linear behavior over $\sqrt{n}$ but with larger values. These increased effective masses for holes and electrons correspond to Fermi velocities of $0.66 \times 10^{6}$ and $0.81 \times 10^{6} \mathrm{~m} / \mathrm{s}$ (fits are shown as black dotted lines). This reduction in the Fermi velocity in twisted samples has been theoretically predicted, ${ }^{15}$ especially for small rotation angles. Small reductions have been measured via Raman spectroscopy ${ }^{17}$ on folded samples and reductions with values down to $70 \%$ have been observed in epitaxial graphene with a single conducting layer. ${ }^{18}$ The here measured strong reduction in the bottom layer confirms the assumption of a rotational stacking fault between the two layers of the folded system.

Using the obtained mobilities and cyclotron masses as extracted from our data, the transport scattering times corresponding to the long-range scatterers are calculated using $\tau_{t}=m_{c} \mu^{*} / e$. Due to the larger mobilities the transport scattering times for the upper layer are larger by a factor of 2.4 for holes and 2.6 for electrons in relation to the ones for charge carriers in the bottom layer. Here, for the upper layer, cyclotron masses according to single monolayer Fermi velocity have been used. Note that a possible underestimation of these masses would imply even higher transport scattering rates and also higher values for the quantum scattering times calculated later on. The dependence of the $\mathrm{SdH}$ oscillations on magnetic field is described by another scattering time, the quantum scattering time $\tau_{q}$. At $1.5 \mathrm{~K}$ and a fixed back-gate voltage the amplitudes are analyzed for different magnetic fields and additionally fitted to the Dingle factor $\exp \left(-\pi m_{c} / e B \tau_{q}\right)$ to calculate these quantum scattering times. ${ }^{19}$ The obtained values are shown in Fig. 5 in dependence of the carrier density and compared to the transport scattering times shown as lines. The quantum scattering times show values on the order of 25-70 fs for holes and electrons increasing with larger carrier density. Extrapolating the values obtained for the bottom layer down to small carrier concentrations being comparable to the ones in the top layer it becomes clear that quantum scattering times are larger in the top one. We assume that the upper layer is more independent on the substrate and screened from possible scattering sources. This explains why both the transport and the quantum scattering times are larger in the upper layer with respect to the bottom one.

In contrast to the high carrier concentration regime where a strong temperature dependence in the longitudinal resistance can only be observed for applied magnetic field, a monotonic and almost linear decrease in the maximum in

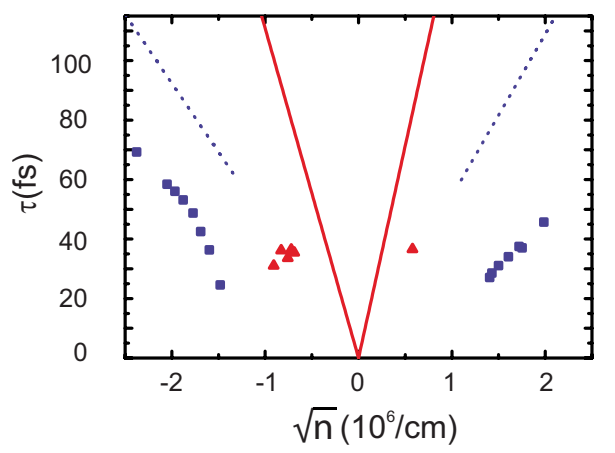

FIG. 5. (Color online) Quantum scattering times in the bottom (top) layer, shown as squares (triangles) over $\sqrt{n}$. Negative values of $\sqrt{n}$ correspond to holes. The transport scattering times are calculated from the experimentally determined mobilities and masses and shown as dotted (bottom layer) and straight (upper layer) lines.

resistivity $\rho_{\max }$ by up to $15 \%$ is observed at $B=0 \mathrm{~T}$ while increasing the temperature from 1.5 to $50 \mathrm{~K}$. This also persists at high magnetic fields ruling out weak localization as a possible reason. To understand this nonmetallic behavior of the decoupled monolayer system it has to be compared to measurements on single monolayers with and without the influence of the substrate. While monolayer devices on silicon dioxide show no significant temperature dependence close to the neutrality point, ${ }^{16}$ a decrease in the maximal resistivity has been observed in suspended graphene. ${ }^{20}$ This behavior is attributed to a very low charge-carrier density near the neutrality point due to less electron and hole puddles in such samples. Theoretical calculations on the temperaturedependent screening of Coulomb disorder ${ }^{21}$ predict that if the charge-carrier density is small enough, a nonmetallic behavior is expected, which explains the mentioned measurements on suspended graphene. We attribute the observed temperature dependence of our sample to a very low carrier concentration at the charge-neutrality point for the upper layer, since this one exhibits properties comparable to suspended samples. The upper layer has a reduced substrate contact, and increased mobilities and scattering times, suggesting low inhomogeneity and therefore less charge carriers near the neutrality point as assumed for suspended samples. ${ }^{22}$

In summary, electronic transport in decoupled graphene monolayers has been studied in detail, showing properties quite different from single-crystal monolayer and bilayer systems. Both layers act as single monolayers conducting in parallel and both show magneto-oscillations with a Berry's phase of $\pi$. The rotational stacking fault of the two layers gives rise to reduced Fermi velocities down to $66 \%$ of the monolayer value. Due to the stacking, the top layer is screened from the substrate and thus has lower carrier concentrations. The majority charge carriers in this screened layer exhibit increased mobilities and scattering times. A nonmetallic temperature dependence additionally indicates the unique nature of this system.

The authors thank V. I. Fal'ko and E. McCann for discussions. This work was financially supported by the excellence cluster QUEST within the German Excellence Initiative. 
${ }^{1}$ K. S. Novoselov, A. K. Geim, S. V. Morozov, D. Jiang, Y. Zhang, S. V. Dubonos, I. V. Grigorieva, and A. A. Firsov, Science 306, 666 (2004).

${ }^{2}$ A. K. Geim and K. S. Novoselov, Nature Mater. 6, 183 (2007).

${ }^{3}$ A. K. Geim, Science 324, 1530 (2009).

${ }^{4}$ Y. Zhang, Y. W. Tan, H. L. Stormer, and P. Kim, Nature (London) 438, 201 (2005).

${ }^{5}$ E. McCann and V. I. Fal'ko, Phys. Rev. Lett. 96, 086805 (2006).

${ }^{6}$ H. Schmidt, T. Lüdtke, P. Barthold, E. McCann, V. I. Fal'ko, and R. J. Haug, Appl. Phys. Lett. 93, 172108 (2008).

${ }^{7}$ P. Poncharal, A. Ayari, T. Michel, and J.-L. Sauvajol, Phys. Rev. B 78, 113407 (2008).

${ }^{8}$ A. Sagar, E. J. H. Lee, K. Balasubramanian, M. Burghard, and K. Kern, Nano Lett. 9, 3124 (2009).

${ }^{9}$ Y. E. Lozovik and A. A. Sokolik, JETP 87, 55 (2008).

${ }^{10}$ C.-H. Zhang and Y. N. Joglekar, Phys. Rev. B 77, 233405 (2008).

${ }^{11}$ H. Min, R. Bistritzer, J.-J. Su, and A. H. MacDonald, Phys. Rev. B 78, 121401(R) (2008).

${ }^{12}$ F. Dolcini, D. Rainis, F. Taddei, M. Polini, R. Fazio, and A. MacDonald, Phys. Rev. Lett. 104, 027004 (2010).

${ }^{13}$ J. Hass, F. Varchon, J. E. Millan-Otoya, M. Sprinkle, N. Sharma,
W. A. de Heer, C. Berger, P. N. First, L. Magaud, and E. H. Conrad, Phys. Rev. Lett. 100, 125504 (2008).

${ }^{14}$ NGS Naturgraphit GmbH.

${ }^{15}$ J. M. B. Lopes dos Santos, N. M. R. Peres, and A. H. Castro Neto, Phys. Rev. Lett. 99, 256802 (2007).

${ }^{16}$ S. V. Morozov, K. S. Novoselov, M. I. Katsnelson, F. Schedin, D. C. Elias, J. A. Jaszczak, and A. K. Geim, Phys. Rev. Lett. 100, 016602 (2008).

${ }^{17}$ Z. Ni, Y. Wang, T. Yu, Y. You, and Z. Shen, Phys. Rev. B 77, 235403 (2008).

${ }^{18}$ W. A. de Heer, C. Berger, X. Wu, P. N. First, E. H. Conrad, X. Li, T. Li, M. Sprinkle, J. Hass, M. L. Sadowski, M. Potemski, and G. Martinez, Solid State Commun. 143, 92 (2007).

${ }^{19}$ X. Hong, K. Zou, and J. Zhu, Phys. Rev. B 80, 241415(R) (2009).

${ }^{20}$ K. I. Bolotin, K. J. Sikes, J. Hone, H. L. Stormer, and P. Kim, Phys. Rev. Lett. 101, 096802 (2008).

${ }^{21}$ E. H. Hwang and S. Das Sarma, Phys. Rev. B 79, 165404 (2009).

${ }^{22}$ K. I. Bolotin, K. J. Sikes, Z. Jiang, M. Klima, G. Fudenberg, J. Hone, P. Kim, and H. L. Stormer, Solid State Commun. 146, 351 (2008). 